\title{
Dissociating location-specific inhibition and attention shifts: Evidence against the disengagement account of contingent capture
}

\author{
Brian A. Anderson • Charles L. Folk
}

Published online: 7 June 2012

(C) Psychonomic Society, Inc. 2012

\begin{abstract}
The study of attentional capture has provided a rich context for assessing the relative influence of top-down and bottom-up factors in visual perception. Some have argued that attentional capture by a salient, irrelevant stimulus is contingent on top-down attentional set (e.g., Folk, Remington, \& Johnston, Journal of Experimental Psychology: Human Perception and Performance 18:1030-1044, 1992). Others, however, have argued that capture is driven entirely by bottom-up salience and that top-down factors influence the postallocation speed of disengagement from the irrelevant stimulus (e.g., Theeuwes, Acta Psychologica 135:77-99, 2010a). In support of this speed-of-disengagement hypothesis, recent findings from the modified spatial-cuing paradigm show that cues carrying a no-go target property produce reverse, or negative, cuing effects, consistent with inhibition of the cue location from which attention has been very quickly disengaged (Belopolsky, Schreij, \& Theeuwes, Perception, \& Psychophysics, 72, 326-341, 2010). Across six experiments, we show that this inhibitory process can be dissociated from shifts of spatial attention and is, thus, not a reliable marker of capture. We conclude that the data are inconsistent with the predictions of the disengagement hypothesis.
\end{abstract}

Keywords Selective attention - Attentional capture · Disengagement $\cdot$ Inhibition $\cdot$ Salience

\footnotetext{
B. A. Anderson ( $\triangle)$

Johns Hopkins University,

Baltimore, MD, USA

e-mail: bander33@jhu.edu

C. L. Folk

Villanova University,

Villanova, PA, USA
}

The visual perceptual system is routinely confronted with more information than can be processed efficiently at a single moment. Indeed, only a fraction of what we experience ever reaches perceptual awareness (Mack \& Rock, 1998). Selective attention refers to the hypothetical mechanism by which limited attentional resources are allocated across the visual field, allowing for the biased representation of selected stimuli (Desimone \& Duncan, 1995). Attention can be allocated either voluntarily to relevant locations or objects (e.g., Posner, 1980) or involuntarily to salient stimuli (e.g., Yantis \& Jonides, 1984). The latter mode of attention allocation has been termed attentional capture, and the mechanisms that underlie it have long been the topic of debate.

\section{Salience-driven attentional capture}

One influential account of attentional capture predicts that involuntary attention allocation is determined solely by local feature contrast (Theeuwes, 1991, 1992, 1994). According to this salience-driven account, the most physically salient stimulus in a display captures attention independently of ongoing task goals. That is, task goals are reflected only in voluntary and strategic attention allocation and deallocation, while attentional capture is impervious to the influence of top-down control at the level of initial selection.

Evidence for a salience-driven account comes primarily from the additional singleton paradigm, in which participants search for a salient target stimulus in a multielement array (e.g., a green circle among green diamonds). On a certain proportion of the trials, a salient distractor (e.g., a red diamond) is presented simultaneously with the target and is thought to compete with the target for attention. When the distractor is more physically salient than the target, response 
time (RT) in target identification is lengthened by its presence (Theeuwes, 1992, 1994). Such an effect has been interpreted as reflecting the capture of attention by the salient, irrelevant singleton and the time needed to shift attention from the location of the distractor to the location of the target. However, when the distractor is less salient than the target, the presence of the distractor does not significantly influence performance (Theeuwes, 1992), consistent with the more salient target capturing attention over the less salient distractor.

\section{Goal-driven attentional capture}

In contrast to a salience-driven account, it has been argued that attentional capture by salient stimuli depends critically on task goals. According to the contingent attentional capture theory, the attention allocation system can be flexibly "set" to respond only to stimuli carrying task-relevant features. Thus, the degree to which a given salient stimulus captures attention is determined by the match between the stimulus and goalrelated top-down attentional control settings (Folk \& Remington, 1998; Folk, Remington, \& Johnston, 1992; Folk, Remington, \& Wright, 1994). By this account, physically salient but goal-irrelevant stimuli are not involuntarily selected by attention.

Evidence for the contingent attentional capture theory comes primarily from the modified spatial-cuing paradigm, in which participants perform a speeded identity judgment on a target stimulus that is preceded in time by a spatially nonpredictive cue. Since the cue provides no information concerning the upcoming target location, participants have no incentive to attend to the cue, and doing so is explicitly discouraged in task instructions. Shorter RTs for targets appearing at the cued location rather than at an uncued location (i.e., a cuing effect) is assumed to reflect the presence of attention at the cued location at the time the target display is presented. Consistent with contingent attentional capture, cues that share a defining feature in common with the target have been shown to selectively produce a cuing effect (Folk \& Remington, 1998; Folk et al., 1992; Folk et al., 1994). For example, Folk et al. (1992) found that when a target defined by onset was searched for, uninformative onset cues produced a cuing effect, whereas color cues did not. In contrast, when a target defined by color was searched for, color cues produced a cuing effect, and onset cues did not.

\section{Attempts to reconcile differences between the paradigms}

Results from the additional singleton paradigm and the spatial-cuing paradigm support very different accounts of attentional capture and, without further qualification, are mutually incompatible (see Theeuwes, 2010a, for a critical review of the literature surrounding this debate). The viability of the salience-driven perspective is dependent on its ability to explain contingent cuing effects in the spatialcuing paradigm, and the viability of the contingent capture perspective is dependent on its ability to account for singleton distractor effects in the irrelevant-singleton paradigm. With respect to the latter, proponents of the contingent capture perspective have relied on the notion of search mode (Bacon \& Egeth, 1994), whereas with respect to the former, proponents of the salience-driven perspective have relied on the notion of speed of disengagement. We will address each in turn, focusing in particular on the speed-of-disengagement hypothesis.

Search mode In the additional singleton paradigm, a salient, irrelevant singleton from one feature dimension (e.g., a red element among green elements) produces a cost in RTs for targets defined in a different dimension (e.g., a circle among diamonds). This result is problematic for the contingent capture perspective in that, if observers are "set" for the shape target, color distractors should not capture attention. However, Bacon and Egeth (1994) found that distraction by the irrelevant color singleton was eliminated when the shape target was presented among heterogeneous nontarget shapes. They argued that under such conditions, observers are forced to search for a particular shape (i.e., the circle), thereby ensuring that observers adopt what they referred to as feature search mode. In contrast, when the target is consistently defined as a shape singleton, observers adopt singleton detection mode, in which the system is set to respond to singletons in general, regardless of the feature dimension in which the singletons are defined. Further evidence for the influence of search mode on performance in this task comes from studies showing that the costs associated with the irrelevant singleton can be predicted from past experience with the different search modes (Leber \& Egeth, 2006a, 2006b), as well as variations in pretrial brain activity assumed to reflect the strength of top-down control settings (Leber, 2010). Thus, from a contingent attentional capture perspective, distraction by irrelevant singletons in the additional singleton paradigm depends on whether the system is configured to respond to particular features or to singletons in general. It should be noted, however, that the notion of search modes is not without controversy (Theeuwes, 2004, 2010a).

Speed of disengagement In the modified spatial-cuing paradigm, cues that do not match the defining properties of the target produce no evidence of attentional capture (i.e., no cuing effect). This result is problematic for the saliencedriven perspective because, at the time the cue appears, it is the most salient item in the display and should, therefore, capture attention regardless of top-down set. To account for 
this result, Theeuwes and colleagues have proposed the speed-of-disengagement hypothesis. According to this notion, attention allocation is initially salience driven such that the most salient stimulus always captures attention. However, the relationship between the features of the stimulus that captures attention and the top-down goals of the observer determines the speed with which attention is then disengaged from the irrelevant stimulus. In the spatial-cuing paradigm, when the cue property does not match the target property, attention is able to quickly disengage and reorient back to fixation prior to the presentation of the target display, such that no cuing effects are observed. Thus, from this perspective, the lack of cuing effects in the spatial-cuing paradigm reflects the influence of top-down attentional control settings on the speed of disengagement, rather than the initial allocation of attention.

To date, evidence in support of the speed-of-disengagement hypothesis is scant. In fact, electrophysiological studies using the N2pc component of the event-related potential (ERP) waveform as a marker of attentional shifts have consistently failed to find any evidence that attention is shifted to nonmatching cues in the modified spatial-cuing paradigm (e.g., Eimer \& Kiss, 2008, 2010; Lien, Ruthruff, Goodin, \& Remington, 2008). However, in two recent articles, the magnitude and direction of cuing effects have been shown to vary quite systematically with the relationship between the cue and the target (Anderson \& Folk, 2010; Belopolsky, Schreij, \& Theeuwes, 2010). This variation has been used as evidence that differences in the cuing effects observed in the spatialcuing paradigm do not reflect which stimuli selectively capture attention but, instead, reflect the speed by which saliencedriven capture is disengaged from the cue (Theeuwes, 2010a, 2010b). Here, we discuss the findings of these two studies, the disengagement hypothesis that has been forwarded to explain these results, and an alternative account in which top-down contingencies exclusively guide attention allocation.

\section{Variation in the magnitude and direction of cuing effects}

Belopolsky et al. (2010) used a spatial-cuing paradigm similar to that in Folk et al. (1992), in which abrupt onset and color targets were paired with onset and color cues. However, the defining feature of the target (i.e., color or abrupt onset) varied from trial to trial. In the critical experiment for the present purposes, participants were informed, on a trial-by-trial basis, to respond only if one type of target appeared and to withhold a response if the other type of target appeared. For example, on a given trial, participants might be instructed to respond to onset targets and withhold a response to color targets. With this go/no-go manipulation, participants were strongly encouraged to apply top-down control over attentional selection, if possible. The results showed that cues matching the go target property produced strong cuing effects indicative of capture. Most important, they found that cues matching the no-go property produced large negative cuing effects, such that RTs on valid trials were much longer than RTs on invalid trials (see also Folk \& Remington, 2008; Lamy, Leber, \& Egeth, 2004). The authors concluded that this negative cuing effect is consistent with the very fast disengagement of attention from cues carrying the no-go property, followed by the application of inhibition specific to the location of the cue, which will be referred to as location-specific inhibition.

In another study using the modified spatial-cuing paradigm, Anderson and Folk (2010) manipulated the perceptual similarity between the cue and a color-defined target. Participants searched for either a red or a green target, the presentation of which was preceded by color cues that varied in terms of the percentage of target color they contained (i.e., perceptual similarity to the target). The magnitude of the cuing effect produced by the different color cues varied linearly with the degree to which they matched the target in color. Furthermore, this variation conformed well to a model in which the differences in the magnitude of the cuing effect were consistent over trials, reflecting varying degrees of involuntary attention allocation, rather than variations in the proportion of trials on which attention was or was not captured by the cues in an all-or-none fashion.

\section{The speed-of-disengagement hypothesis and its predictions}

Theeuwes (2010a) has argued that the two studies described above provide strong evidence that top-down control settings affect the speed of disengagement from irrelevant cues, rather than the initial allocation of attention. Specifically, according to the speed-of-disengagement account, the variation in the cuing effects reported by Anderson and Folk (2010) reflect disengagement from captured attention that is increasingly slower the more the cue looks like the target. Essentially, it takes participants more time to realize that the eliciting stimulus is different from the target with increasing perceptual similarity, resulting in larger cuing effects (Theeuwes, 2010a). Furthermore, Theeuwes (2010a) argued that the negative cuing effects reported by Belopolsky et al. (2010) reflect a form of suppression akin to inhibition of return (IOR). Specifically, the no-go manipulation provides participants with a strong incentive to avoid processing stimuli possessing a feature in common with the no-go target stimulus, resulting in the faster disengagement of attention. When disengagement is fast enough, there is time to initiate the inhibition of the formerly attended location prior to the appearance of the target display (Belopolsky et 
al., 2010). Thus, according to a disengagement account, the magnitude and direction of cuing effects is reflective of the speed with which the system recovers from salience-driven capture.

To more clearly illustrate the relationship between speed of disengagement and the nature of cuing effects as described by Belopolsky et al. (2010) and Theeuwes (2010a), consider Fig. 1. Lines a, b, and c represent the predicted effects of cue validity for slow, fast, and very fast disengagement speeds, respectively. When the defining property of the cue matches the defining property of the target, disengagement speed will be slow, such that attention will still be at the cued location when the target display arrives, resulting in a large positive cuing effect, with short RTs on valid trials and long RTs on invalid trials (line a). When the cue property is very different from the target property, however, disengagement will be fast enough that recovery from capture (illustrated by the solid arrows) is complete by the time the target display appears, resulting in no observable cuing effect (line b). When the cue does not match the target and is additionally associated with a no-go property, disengagement is very fast, resulting not only in recovery from capture (solid arrows), but also in the application of inhibition (IOR) to the cued location prior to the appearance of the target display (illustrated by the dashed arrow). Under these conditions, there is a selective elevation of RTs on valid trials because the target later appears at the location to which inhibition has been applied, resulting in a negative cuing effect (line c). It is important to note that our use of the categorical terms slow, fast, and very fast are defined relative to the cue-target stimulus onset asynchrony (SOA). If the time to disengage exceeds the cue-target SOA, disengagement is considered slow. If the time to disengage is less than the cue-target SOA, disengagement is considered fast. If the time to disengage is so much less than the cue-target SOA that there is time to apply inhibition to the cued location before the target appears, disengagement is considered very fast.

\section{An alternative to the speed-of-disengagement hypothesis}

Although the results of Anderson and Folk (2010) and Belopolsky et al. (2010) are consistent with the speed-ofdisengagement account, they are also consistent with an account in which top-down control settings affect initial attentional allocation, rather than postallocation disengagement speed. According to this perspective, the cuing effects consistent with slow disengagement (Fig. 1, line a) reflect attention that has, in fact, been captured, while cuing effects consistent with fast disengagement (Fig. 1, line b) reflect attention that was never captured (as opposed to captured attention that has recovered). Intermediate variations in the

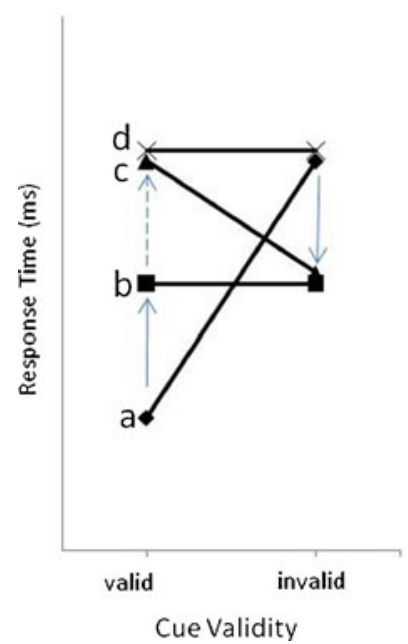

Fig. 1 Emprirical signatures of attentional capture. According to the speed-of-disengagement account, (a) reflects slow disengagement, (b) reflects fast disengagement, as illustrated by the solid arrows, and (c) reflects very fast disengagement (solid arrows) with the addition of location-specific inhibition (dashed arrow). According to the contingent attentional capture theory, (d) and (a) reflect captured attention with and without location-specific inhibition, while (c) and (b) reflect uncaptured attention with and without location-specific inhibition, respectively

magnitude of capture as a function of cue-target similarity reflect variations in the amount of resources that are captured. Most critically for the purposes of the present study, it is possible that the location-specific inhibition (i.e., negative cuing effects) found for cues containing a no-go property (Fig. 1, line c) reflects a form of suppression that is dissociable from the capture of attention. For example, Lamy et al. (2004) found negative cuing effects associated with uninformative cues that did not match the target property but interpreted these effects as a form of top-down feature-based suppression that serves to prevent the capture of attention by the cue. In addition, Sawaki and Luck (2010) demonstrated ERP evidence of the suppression of a salient distractor in the absence of the N2pc (an ERP marker of attentional capture), regardless of whether the distractor appeared in an attended or unattended region of space. Thus, the negative cuing effects observed by Belopolsky et al. may reflect topdown, feature-based suppression that is applied independently of whether attention was captured and disengaged from the cues or not. We will refer to this alternative as the capture-independent inhibition account.

Given the important role variations in the magnitude and direction of cuing effects have played in the debate over the role of top-down set in attentional capture, the present study was designed to adjudicate between the two possible accounts outlined above, with particular emphasis on the interpretation of the negative cuing effects reported by Belopolsky et al. (2010). The logic of our approach is as follows. If the location-specific inhibition associated with 
cues containing no-go properties is a direct consequence (and therefore a marker) of the capture and very fast disengagement of attention, it should occur only when attention is indeed captured, and it should always take the form of negative cuing effects, as illustrated in Fig. 1, line c. This, according to the disengagement hypothesis, is because the inhibition is possible only if attention is disengaged very quickly, which would therefore necessarily yield relatively short RTs on invalid trials. If, however, the location-specific inhibition associated with no-go cues is independent of the capture and very fast disengagement of attention, it should occur regardless of whether attention has been shifted to the cued location or not, and it should be evident even when capture is produced by a cue that is similar enough to the target that fast or very fast disengagement is not possible. That is to say, by our capture-independent inhibition account, attentional capture and location-specific inhibition can manifest simultaneously and are not mutually exclusive, as is predicted by the speed-of-disengagement account.

Most important, under conditions in which attentional capture and location-specific inhibition co-occur, a specific empirical signature quite unlike any predicted by the disengagement hypothesis should obtain. Specifically, as is illustrated in Fig. 1, line d, RTs should be relatively long not only on invalid trials, which reflects the capture of attention and lack of disengagement, but also on valid trials, which reflects the application of location-specific inhibition that is applied independently of any disengagement (note that by this account, the application of location-specific inhibition to the cued location also occurs on invalid trials but is not observable because, by definition, the target does not appear at the cued location on invalid trials). ${ }^{1}$ In the experiments that follow, we replicate and extend the evidence that has been used to argue for the speed-of-disengagement hypothesis and then provide evidence that is uniquely consistent with the capture-independent inhibition account in which suppression is independent of the allocation of spatial attention.

\section{Experiment 1}

Experiment 1 was intended to replicate evidence that has been used to argue in favor of the speed-of-disengagement

\footnotetext{
${ }^{1}$ It is important to note that Fig. 1 represents a somewhat simplified schematic and that the predicted patterns are fundamentally relational in nature (as indicated by the arrows). For example, when lines a and $d$ are compared, what is important is that the data points be equivalent on invalid trials (since attention needs to be reoriented in both cases) and that RTs are longer on valid trials for line d (since inhibition occurs for line $d$ but not line a); the exact position of the data point for line $d$ on valid trials depends upon the degree to which inhibition overshadows any benefit of attentional biasing due to the capture of attention.
}

hypothesis. To this end, we tested participants in a version of the modified spatial-cuing paradigm in which the participants searched for color-defined targets that were either red or blue, which were preceded unpredictably by red, green, or blue cues. Participants reported the identity of red targets while withholding a response to blue targets. The speed-ofdisengagement hypothesis predicts that all three color cues will capture attention in this context but that the speed of disengagement should vary. Specifically, disengagement from the red cues (which match the go target color) should be slow, resulting in large cuing effects, whereas disengagement from green cues (which are perceptually distinct from the target colors) should be fast, resulting in no cuing effects. Most important, disengagement from blue (i.e., nogo-colored) cues should be very fast, allowing the application of location-specific inhibition, resulting in negative cuing effects. This pattern would conform to the empirical predictions of the speed-of-disengagement hypothesis but also would ensure that our paradigm is sensitive to the entire range of disengagement speeds that it predicts.

\section{Method}

Participants Twenty-two undergraduate and graduate students participated in this experiment. Undergraduate students were recruited from the Villanova University human participant pool. All were screened for normal or correctedto-normal visual acuity and color vision. Undergraduate students were compensated for their time with credit toward fulfillment of a class research requirement, and graduate students were compensated with $\$ 10$.

Apparatus A Zenith 386 microcomputer equipped with a Sigma Design, Color 400 graphics board was used to present the stimuli on a Princeton Graphics Ultrasync monitor. Participants viewed the monitor from a distance of $50 \mathrm{~cm}$ through lensless goggles attached to a porthole in the front of a viewing box. The inside of the box was painted black, and all but the screen of the monitor was occluded when participants peered through the goggles.

Stimuli Each trial involved three different displays. An example of each, along with their sequence of presentation and time course, is shown in Fig. 2. The first display, the fixation display, consisted of a fixation square $\left(0.34^{\circ} \times 0.34^{\circ}\right.$ of visual angle) surrounded by four peripheral boxes $\left(1.15^{\circ} \times 1.15^{\circ}\right)$ placed $4.1^{\circ}$ above, below, to the left, and to the right of fixation. The color of the boxes and fixation square was light gray (RGB: $85,85,85$; CIE: $x=.35, y=.36$ ), and the background of the CRT screen was black.

The second display, the cue display, consisted of the appearance of four sets of small circles $\left(0.23^{\circ}\right.$ in diameter $)$ surrounding each of the four peripheral boxes in a diamond 


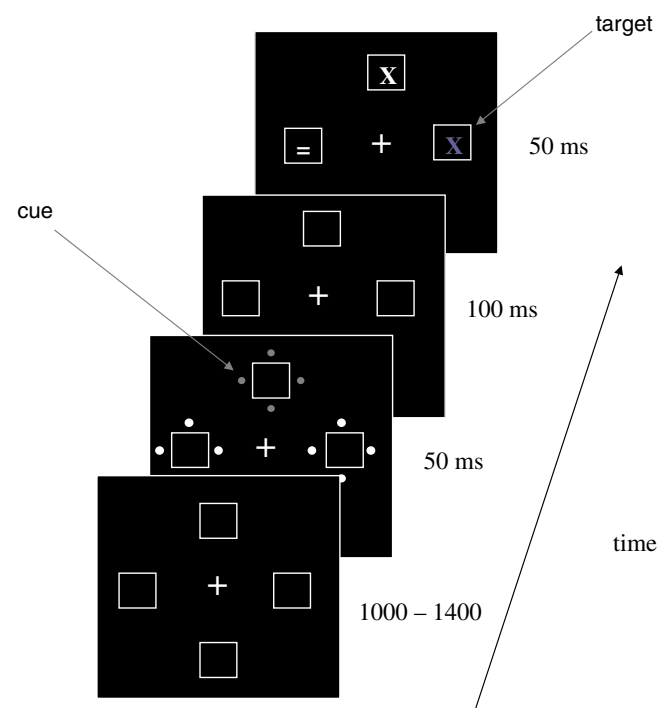

Fig. 2 Sequence of events and time course of a trial

configuration. Three sets of the circles were white (RGB: 255, 255, 255; CIE: $x=.35, y=.36$ ), while the other set was one of three possible colors. The colors used were red (RGB: 255, 85, 85; CIE: $x=.56, y=.34$ ), green (RGB: 85, 255, 85; CIE: $x=.33, y=.55$ ), and blue (RGB: 85, 85, 255; CIE: $x=.20, y=.13$ ).

The final display, the target display, consisted of the appearance of an "X" or an "=" in each of the peripheral boxes. These characters subtended approximately $0.57^{\circ}$ of visual angle in height and width. Like the circles, three of the characters were white, while the other was colored. Targets were either red or blue.

Design The experiment consisted of four blocks of 96 trials. Within each block, all cue colors and target colors were presented equally often. Each target character (i.e., colored $\mathrm{X}$ or $=$ ) appeared equally often at each of the four possible locations within each condition, while the identity of the nontarget characters were chosen randomly on each trial. The location of the cue circles was determined randomly on each trial, with the constraint that for each cue-color-targetcolor combination, the cue appeared at the same location as the target on $25 \%$ of the trials and at a different location on $75 \%$ of the trials within a block.

Procedure Each participant was tested individually over the course of a single 50-min session. Participants were instructed to respond as quickly as possible while minimizing errors and to withhold responses to blue targets. Maintaining fixation on the central square was highly stressed, and participants were told that failing to do so would impair overall performance. Participants were also fully informed of the relationship between cue location and target location and were told to try to ignore the cue.
Each trial began with a 500-ms-long presentation of the fixation display. After this $500-\mathrm{ms}$ period, the fixation square blinked off for $100 \mathrm{~ms}$ and then back on for a randomly varying foreperiod of $1,000,1,100,1,200$, or $1,400 \mathrm{~ms}$. The cue display then appeared for $50 \mathrm{~ms}$, followed by the fixation display for $100 \mathrm{~ms}$. Finally, the target display appeared for $50 \mathrm{~ms}$, followed again by the fixation display. The next trial sequence was initiated $1,000 \mathrm{~ms}$ after a response had been made or the trial had terminated. Phenomenally, the four display boxes and fixation square appeared to remain on the CRT screen for the duration of each trial and intertrial interval. Contamination of RTs by eye movement was unlikely, given the fact that the SOA between the cue and the target was $150 \mathrm{~ms}$.

Participants made a target identification by pressing the "." key with their right index finger and the "0" key with their left index finger on the numeric keypad for the " $\mathrm{X}$ " and "=" targets, respectively. RT was measured from the onset of the target display until a response was made or $1,500 \mathrm{~ms}$ had passed. If a response was not made in this $1,500-\mathrm{ms}$ period, the trial was terminated. The computer emitted a 500 -mslong $1000-\mathrm{Hz}$ tone to inform the participant when an error had been made. Error trials were followed by a buffer trial, the parameters of which were randomly drawn from the set for that block. RTs for error or buffer trials were not included in the analysis.

\section{Results}

Mean correct RTs for valid and invalid trials, as a function of cue color, are shown in Fig. 3. The data were subjected to a $3 \times$ 2 analysis of variance (ANOVA), with cue color (red, green, and blue) and cue validity (valid and invalid) as withinsubjects variables. A main effect of cue validity, $F(1,21)=$ 4.68, $p<.05, \eta_{\mathrm{p}}{ }^{2}=.18$, and an interaction between cue validity and cue color, $F(2,42)=18.10, p<.0001, \eta_{\mathrm{p}}{ }^{2}=$ .46 , were observed. Simple-effects analyses of cue validity at each cue color revealed a significant positive cuing effect for red (go color) cues, $F(1,21)=24.78, p<.0001, \eta_{\mathrm{p}}{ }^{2}=.54$, a significant negative cuing effect for blue (no-go color) cues, $F(1,21)=8.67, p<.01, \eta_{\mathrm{p}}{ }^{2}=.29$, and no significant effect of validity for green cues, $F<1$. Given the theoretical significance of the effect of the different cues within each validity condition (i.e., the arrow paths in Fig. 1), simple effects of cue color at each level of cue validity were conducted. For valid trials, cue color produced a significant effect, $F(2,42)=10.06$, $p<.001, \eta_{\mathrm{p}}{ }^{2}=.32$, and pairwise comparisons (Tukey test, $\alpha=.05$ ) showed that RTs for red cues were significantly shorter than those for both green and blue cues (the difference between the latter two just failed to reach the critical difference). For invalid trials, cue color once again produced a significant effect, $F(2,42)=9.93, p<.001, \eta_{\mathrm{p}}{ }^{2}=.32$, and 


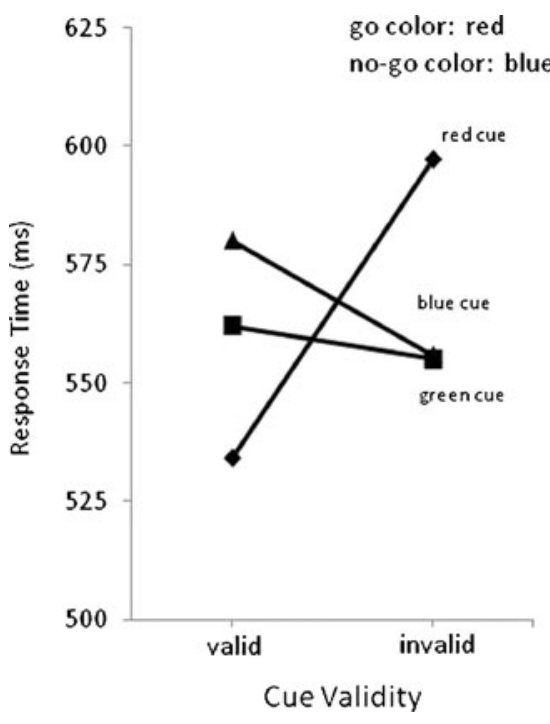

Fig. 3 Mean response time as a function of cue validity and cue color in Experiment 1. Go targets were red, and no-go targets were blue

pairwise comparisons showed that red cues produced significantly longer RTs than did green or blue cues (RTs for the latter two cue conditions did not differ).

A similar ANOVA conducted on error rates revealed only a main effect of validity, $F(1,21)=18.94, p<.001, \eta_{\mathrm{p}}{ }^{2}=.47$. The error rate data are depicted in Table 1.

\section{Discussion}

The results are clear and strikingly resemble the predictions of the speed-of-disengagement hypothesis (Belopolsky et al., 2010). Cues that matched the target color produced large positive cuing effects, cues that did not match the target color produced no cuing effects, and cues carrying the no-go color produced negative cuing effects. Thus, we demonstrate evidence consistent with slow, fast, and very fast disengagement (followed by the application of location-specific inhibition for no-go cues) in the same experiment, as a function of the relationship between the eliciting stimulus and top-down priorities. The pattern of RTs for red and green cues (i.e., cues defined by colors not associated with a no-go target) is consistent with the results of previous studies showing significant cue validity effects for target-colored cues and no cuing effects for non-target-colored cues (e.g., Folk \& Remington, 1998). In addition, the pattern of RTs for blue (i.e., no-go-colored) cues is consistent with the results of Folk and Remington (2008) and Belopolsky et al. (2010) in that significant negative cuing effects were obtained. The overall pattern conforms to the empirical predictions of the speed-of-disengagement hypothesis under the present experimental conditions but also confirms that our paradigm is sensitive to the entire range of predicted disengagement speeds. Note that a general, nonspatial disruption in processing was not associated with the blue no-go target color cue in this paradigm, which would have resulted in generally elevated RTs on both valid and invalid trials for blue cues, relative to green cues.

\section{Experiment 2}

In Experiment 1, we replicated results from previous research concerning the magnitude and direction of cuing effects that have been used to argue in favor of the disengagement hypothesis and extended this research by demonstrating evidence consistent with slow, fast, and very fast disengagement in the same experiment. Experiment 2 was conducted to confirm that the pattern obtained in Experiment 1 is not specific to the particular assignment of colors and to provide further support for the notion that the observed inhibition for the no-go color cue reflects a form of top-down control over selection, as predicted by the captureindependent inhibition account. Anderson and Folk (2010) demonstrated that the influence of top-down control over attentional selection varies linearly with the perceptual similarity between the cue and the current attentional control settings. If location-specific inhibition is similarly influenced by top-down control settings - in this case, for a

Table 1 Error rates (proportion incorrect) as a function of cue condition across Experiments 1-6

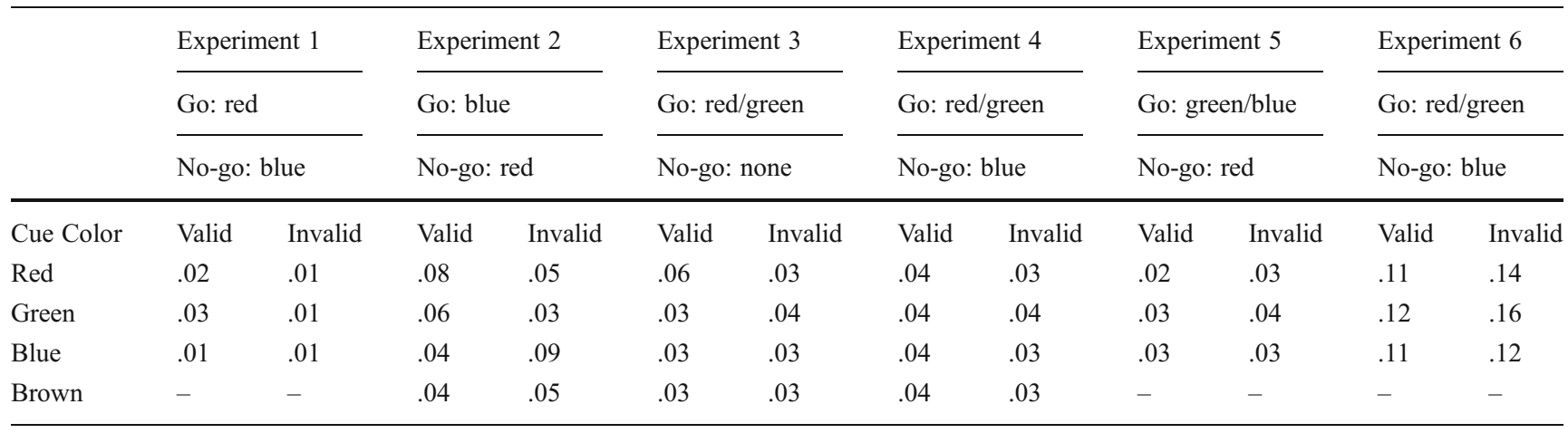


to-be-ignored stimulus feature-the magnitude of the observed inhibition should also vary linearly with the similarity between the cue color and the to-be-ignored no-go target color. To this end, we replicated Experiment 1 with different go and no-go target colors while introducing a blended color cue that looked perceptually similar to, although distinct from, the color of the no-go target. If the inhibition observed in Experiment 1 reflects a form of top-down control over selection, this blended color cue should produce an intermediate degree of inhibition on valid trials.

\section{Method}

Participants Ten undergraduate students were recruited from the Johns Hopkins University human participant pool. All were screened for normal or corrected-to-normal visual acuity and color vision. Participants were compensated for their time with extra credit toward one of several psychology courses. None of the participants had participated in Experiment 1.

Apparatus and stimuli A Mac Mini was used to present the stimuli on a Dell P991 monitor. Participants viewed the monitor from a chinrest at a distance of $50 \mathrm{~cm}$. The color of the boxes and fixation square was white. Cue colors were red (RGB: 255, 0, 0; CIE: $x=.64, y=.33$ ), green (RGB: 0 , 255, 0; CIE: $x=.30, y=.60$ ), blue (RGB: 0, 0, 255; CIE: $x=.15, y=.06$ ), and a blending of red and green (RGB: 222, 192, 0; CIE: $x=.45, y=.48$ ). In all other respects, the apparatus and stimuli were identical to those in Experiment 1.

Design and procedure The design and procedure were identical to those in Experiment 1, with the exception that participants were told to respond to blue targets and withhold responses to red targets. The experiment consisted of a total of 512 trials, with a break every 100 trials (except for the last 112 trials). Additionally, there were no buffer trials, and participants responded using the " $\mathrm{z}$ " and " $\mathrm{m}$ " keys.

\section{Results}

Mean correct RTs for valid and invalid trials as a function of cue color are shown in Fig. 4 . A $4 \times 2$ ANOVA with cue color (red, green, blended, and blue) and cue validity (valid and invalid) as within-subjects variables revealed only an interaction between cue validity and cue color, $F(3,27)=$ $17.64, p<.0001, \eta_{\mathrm{p}}{ }^{2}=.71$. Simple-effects analyses revealed a significant positive cuing effect for blue (go color) cues, $F(1,9)=34.37, p<.001, \eta_{\mathrm{p}}{ }^{2}=.79$, a significant negative cuing effect for red (no-go color) cues, $F(1,9)=14.70$,

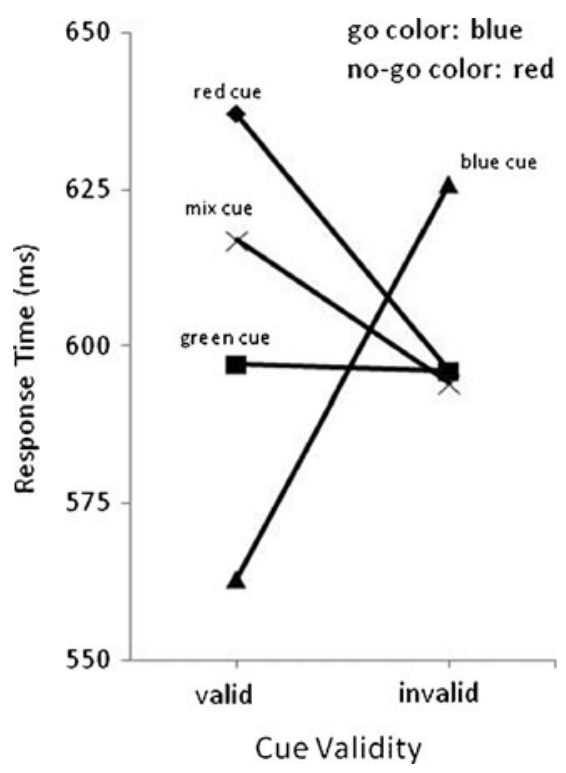

Fig. 4 Mean response time as a function of cue validity and cue color in Experiment 2. Go targets were blue, and no-go targets were red

$p<.01, \eta_{\mathrm{p}}{ }^{2}=.62$, no significant cuing effect for green cues, $\mathrm{F}<1$, and a marginally significant negative cuing effect for blended (perceptually similar to no-go color) cues, $F(1,9)=$ $4.42, p=.064, \eta_{\mathrm{p}}{ }^{2}=.33$. The variation in the magnitude and direction of cuing effects was well accounted for by a linear trend in the predicted direction, $F(1,9)=44.70, p<.0001$, $\eta_{\mathrm{p}}{ }^{2}=.83$. Simple effects of cue color at each level of validity revealed significant effects for both valid and invalid trials, $F(3,27)=7.05, p<.001, \eta_{\mathrm{p}}{ }^{2}=.44$, and $F(3,27)=7.42$, $p<.001, \eta_{\mathrm{p}}{ }^{2}=.45$, respectively. Pairwise comparisons showed that on valid trials, the blue cues differed from the red and blended cues. On invalid trials, blue cues differed from all other cues, which did not differ from one another.

A similar ANOVA was conducted on error rate. This analysis revealed no significant main effects or interactions. The error rate data are depicted in Table 1.

\section{Discussion}

The results of Experiment 2 replicate the results of Experiment 1 very closely, again producing evidence consistent with slow, fast, and very fast disengagement. This outcome precludes the possibility that our results are specific to particular cue colors. Importantly, the blended color cue in the present experiment produced the predicted intermediate level of inhibition on valid trials. This finding extends the findings of Anderson and Folk (2010) to the inhibition of tobe-ignored features, providing additional evidence that location-specific inhibition reflects a form of top-down attentional control, as predicted by the capture-independent inhibition account. However, to the extent that inhibition builds over 
time following the disengagement of attention, variations in the speed of disengagement could also account for this pattern.

\section{Speed of disengagement or capture-independent inhibition?}

Experiments 1 and 2 demonstrate that we can obtain variations in the magnitude and direction of cuing effects that are consistent with the predictions of the speed-of-disengagement hypothesis. However, as was noted in the introduction, this pattern of results is also consistent with the captureindependent inhibition account in which attention is captured only by cues carrying the target-defining color, with negative cuing effects reflecting location-specific inhibition that is under top-down control and applied independently of attention shifts. One way of distinguishing between these two possibilities is to create conditions under which the captureindependent inhibition account also predicts capture by cues associated with a no-go color. Under such conditions, the two accounts make very different predictions with respect to location-specific inhibition effects. Specifically, as was noted in the introduction, the speed-ofdisengagement account predicts one of the patterns shown in Fig. 1, lines $b$ and c, depending on how rapidly attention can be disengaged from the no-gocolored cue. In contrast, the capture-independent inhibition account makes a unique prediction, represented by Fig. 1, line d. In the invalid cue condition, RTs for nogo-colored cues should not differ from those produced by go-colored cues, reflecting the capture of attention to the cued (i.e., nontarget) location. In the valid cue condition, no-go-colored cues should produce an increase in RT relative to go-colored cues, reflecting the application of location-specific inhibition to the cued (i.e., attended target) location. It is important to reiterate that the result predicted by the capture-independent inhibition account is impossible under a speed-ofdisengagement account, which requires that evidence of location-specific inhibition (i.e., an increase in RT relative to go cues on valid trials) be accompanied by evidence of rapid disengagement (i.e., a decrease in RT relative to go cues on invalid trials).

To test these predictions, we introduced a manipulation for which both accounts would predict capture by a cue associated with a no-go color. Specifically, we varied the specific color of the target unpredictably across trials. Previous research has shown that when the specific color of the target is unpredictable, all color singleton cues produce evidence of attentional capture, regardless of whether they match the target color (Folk \& Anderson, 2010). From a speed-of-disengagement perspective, this is because the similarity between the singleton status of the cues and the singleton definition of the target prevents the rapid disengagement of attention. From a contingent capture perspective, this is because color singleton cues match the top-down set for color singleton targets. The important point is that both perspectives predict that under these conditions, cues associated with a no-go color should capture attention. This makes it possible to then distinguish between the predictions of the two accounts with respect to disengagement and location-specific inhibition, as outlined above.

\section{Experiment 3}

Before testing the effects of no-go color cues under conditions in which the target color is unpredictable, it is important to first establish that varying target color unpredictably does indeed produce a top-down set for color singletons in general, resulting in capture by (or slow disengagement from) cues that do not match the target color. Thus, in Experiment 3, targets were unpredictably red or green, and cues were red, green, brown (i.e., red/green blended as in Experiment 2), or blue. The inclusion of both brown and blue cues (i.e., cues that do not match the target colors) provides the critical test of whether participants adopt a top-down set for color singletons in general. If so, then in addition to red and green (i.e., target-colored) cues, these nontarget color cues should also produce evidence of capture, resulting in a cuing effect of similar magnitude.

\section{Method}

Participants Fifteen undergraduate students were recruited from the Villanova University human participant pool. All were screened for normal or corrected-to-normal visual acuity and color vision. Participants were compensated for their time with credit toward fulfillment of a class research requirement. None of the participants had participated in any of the previous experiments.

Apparatus and stimuli The apparatus and stimuli were identical to those used in Experiment 1, with the exception that targets could be red or green and cues could be red, green, brown (RGB: 127, 127, 0; CIE: $x=.42, y=.51$ ), or blue.

Design and procedure The design and procedure were identical to those in Experiment 1, with the exceptions that blue no-go targets were replaced with green go targets and brown cues were added. The experiment consisted of eight blocks of 64 trials, and within each block, the two target colors appeared equally often. 


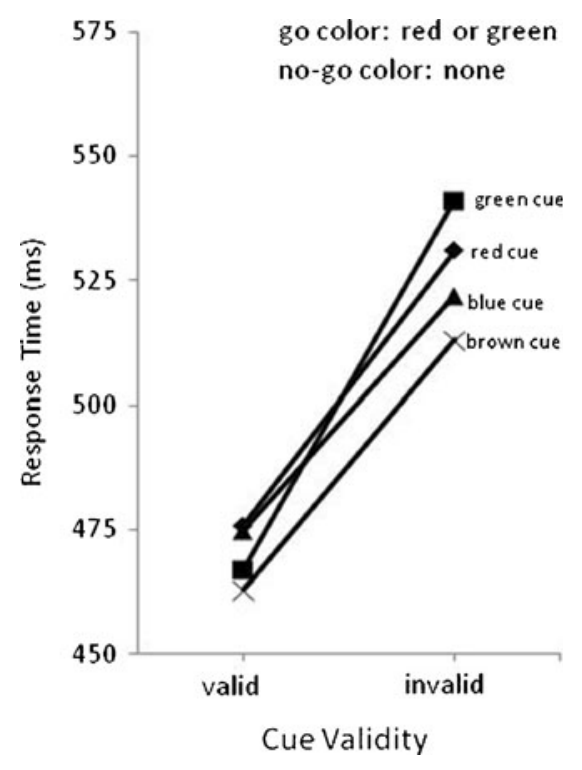

Fig. 5 Mean response time as a function of cue validity and cue color in Experiment 3. Go targets were unpredictably red or green

\section{Results}

Mean correct RTs for valid and invalid trials as a function of cue color are shown in Fig. 5. The data were subjected to a $4 \times 2$ ANOVA, with cue color (red, green, brown, and blue) and cue validity (valid and invalid) as within-subjects variables. The analysis revealed only a significant main effect of cue validity, $F(1,14)=100.87, p<.0001, \eta_{\mathrm{p}}{ }^{2}=.88$.

A similar ANOVA was conducted on error rate. This analysis revealed only a significant main effect of cue validity, $F(1,14)=11.38, p<.01, \eta_{\mathrm{p}}{ }^{2}=.45$. The error rate data are depicted in Table 1.

\section{Discussion}

The results of Experiment 3 show what is immediately evident in Fig. 5: When target color varies unpredictably between red and green, all cue colors produce evidence of attentional capture that is similar in magnitude. This is supported statistically by the main effect of cue validity and the absence of an interaction between cue color and cue validity. Thus, the results confirm that our target uncertainty manipulation encourages a top-down set for color singletons in general and that, under these conditions, cues that do not match the specific target colors nonetheless capture attention.

\section{Experiment 4}

Having established that target uncertainty results in capture by cues that do not match target colors, we are now in a position to introduce the critical no-go manipulation. Experiment 4 was identical to Experiment 3, with the exception that a blue no-go target was included in the design. According to the speed-of-disengagement account, the no-go status of the color blue should selectively increase the speed of disengagement of attention from blue cues. Thus, relative to all the other cue colors, blue cues should show reduced RTs on invalid trials and increased RTs on valid trials, producing a pattern similar to that shown in Fig. 1, lines b or c, depending on the degree to which the speed of disengagement is increased. According to the capture-independent inhibition account, the no-go status of the color blue should result in the application of inhibition to the location of blue cues that is independent of the locus and/or disengagement status of attention. Thus, assuming that attention is indeed captured by blue cues (as suggested by the results of Experiment 3), this account predicts that RTs for blue cues should be no different than those for any other cues on invalid trials (because attention and inhibition have been applied to a cued nontarget location) but significantly longer than those for any other cues on valid trials (reflecting the fact that inhibition has been applied to the blue-cued target location), producing a pattern similar to that shown in Fig. 1, line d.

\section{Method}

Participants Eighteen undergraduate students were recruited from the Villanova University human participant pool. All were screened for normal or corrected-to-normal visual acuity and color vision. Participants were compensated for their time with credit toward fulfillment of a class research requirement. None of the participants had participated in any of the previous experiments.

Apparatus and stimuli The apparatus and stimuli were identical to those used in Experiment 1, with two exceptions. Two go targets were used, which consisted of red and green. In addition, a brown cue was added.

Design and procedure The design and procedure were identical to those in Experiment 1, with the exception that the experiment consisted of six blocks of 96 trials.

\section{Results}

Mean correct RTs for valid and invalid trials as a function of cue color are shown in Fig. 6. The data were subjected to a $4 \times 2$ ANOVA, with cue color (red, green, brown, and blue) and cue validity (valid and invalid) as within-subjects variables. The analysis revealed main effects of both cue validity and cue color, $F(1,17)=27.41, p<.0001, \eta_{\mathrm{p}}{ }^{2}=.62$, and $F(3$, $51)=7.50, p<.001, \eta_{\mathrm{p}}{ }^{2}=.31$, respectively, and an interaction between cue validity and cue color, $F(3,51)=5.84, p<.01$, 


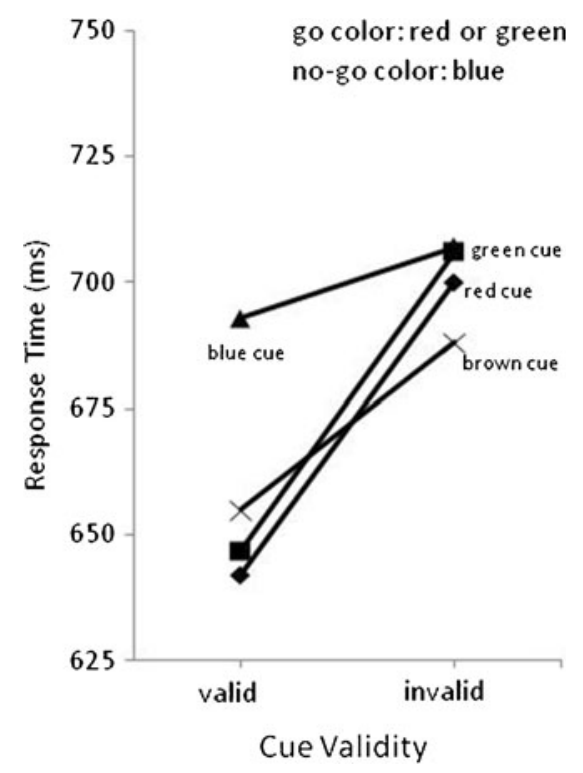

Fig. 6 Mean response time as a function of cue validity and cue color in Experiment 4. Go targets were unpredictably red or green, while nogo targets were blue

$\eta_{\mathrm{p}}{ }^{2}=.26$. Simple effects analyses of the effects of cue validity at each level of cue color revealed significant positive cuing effects for red, green, and brown cues, $F(1,17)=22.37$, $p<.0001, \eta_{\mathrm{p}}^{2}=.57 ; F(1,17)=23.70, p<.0001, \eta_{\mathrm{p}}{ }^{2}=.58$; and $F(1,17)=10.03, p<.01, \eta_{\mathrm{p}}{ }^{2}=.37$, respectively, while the blue (no-go color) cue showed no reliable difference between valid and invalid trials, $F(1,17)=2.95, p>.10$. More important, simple effects of cue color at each level of cue validity revealed no significant effect for invalid trials, $F(3,51)=1.58, p>.20$, but a highly significant effect for valid trials, $F(3,51)=12.53, p<.0001$, $\eta_{\mathrm{p}}^{2}=.42$. Pairwise comparisons for the valid condition revealed a significant difference between blue cues and all other color cues, which did not differ significantly from one another.

A similar ANOVA was conducted on error rate. This analysis revealed no significant main effects or interactions. The error rate data are depicted in Table 1.

\section{Discussion}

The results of Experiment 4 are uniquely in line with the predictions of the capture-independent inhibition account in which top-down control settings determine whether attention is allocated to cues and top-down inhibition associated with no-go color cues operates independently of the allocation of spatial attention. First, the fact that blue cues produced RTs on invalid trials nearly identical to invalid RTs for all other color cues suggests that spatial attention was at the location of the blue cues when the target display appeared. This stands in clear contrast to the results of the first two experiments, in which a robust difference between go and no-go color cues was evident on invalid trials. Second, the significant elevation of RTs on valid trials for blue cues, relative to the other cues, suggests that inhibition was applied to the location of the cue containing the no-go color. In other words, given no evidence of disengagement for blue cues (similarly elevated RTs on invalid trials), the fact that they still show location-specific inhibition (as indicated by the elevation of RT on valid trials for blue cues) indicates that location-specific inhibition can be applied even when attention has not disengaged from the cued location. This clearly violates the predictions of the speedof-disengagement hypothesis.

\section{Experiment 5}

Although the pattern of results in Experiment 4 is consistent with the application of capture-independent inhibition associated with no-go colored cues, the overall RTs for the experiment were noticeably longer than in any of the other experiments. This is not surprising, given the combination of four possible cue colors, three possible target colors, and a go/no-go decision. Nonetheless, one might argue that our conclusions are clouded by a potential ceiling effect that masks a large positive cuing effect indicative of slow disengagement for the blue color cues. Under this account, the significant lengthening of RTs on valid trials, rather than reflecting location-specific inhibition, might instead indicate some sort of nonspatial, general lengthening associated with the appearance of a no-go color (although this seems unlikely, given that there was no evidence of such a general lengthening effect in Experiments 1 and 2). To rule out this possibility and confirm that the results of Experiment 4 were not specific to the particular assignment of colors, we replicated Experiment 4 with two critical modifications. First, the target assignment for blue and red were switched, as in Experiment 2, such that go targets were unpredictably blue or green and no-go targets were red. Second, to reduce overall RTs, the number of possible cue and target locations was reduced from four to two, and the brown cue was omitted from the cue ensemble. A replication of the results of Experiment 4 in this context would not only rule out a possible ceiling effect, but also show that this pattern of results generalizes across different experimental conditions.

Method

Participants Twenty-two undergraduate students were recruited from the Villanova University human participant pool. All were screened for normal or corrected-to-normal 
visual acuity and color vision. Participants were compensated for their time with credit toward fulfillment of a class research requirement. None of the participants had participated in any of the previous experiments.

Apparatus and stimuli The apparatus and stimuli were identical to those used in Experiment 4, with the exception that only the left and right locations were included (nothing ever occurred at the top and bottom locations) and the brown cues did not appear.

Design and procedure The design and procedure were identical to those in Experiment 4, with the exception that cues indicated the target location on $50 \%$ of the trials and a nontarget location on $50 \%$ of the trials. In addition, participants were instructed to respond to targets that were green or blue and to withhold responses to red targets. The experiment consisted of eight blocks of 64 trials.

\section{Results}

Mean correct RTs for valid and invalid trials as a function of cue color are shown in Fig. 7. The data were subjected to a $3 \times 2$ ANOVA, with cue color (blue, green, and red) and cue validity (valid and invalid) as within-subjects variables. The analysis revealed main effects of cue color and cue validity, $F(2,42)=3.96, p<.05, \eta_{\mathrm{p}}{ }^{2}=.16$, and $F(1,21)=9.49$, $p<.01, \eta_{\mathrm{p}}{ }^{2}=.31$, respectively, and an interaction between

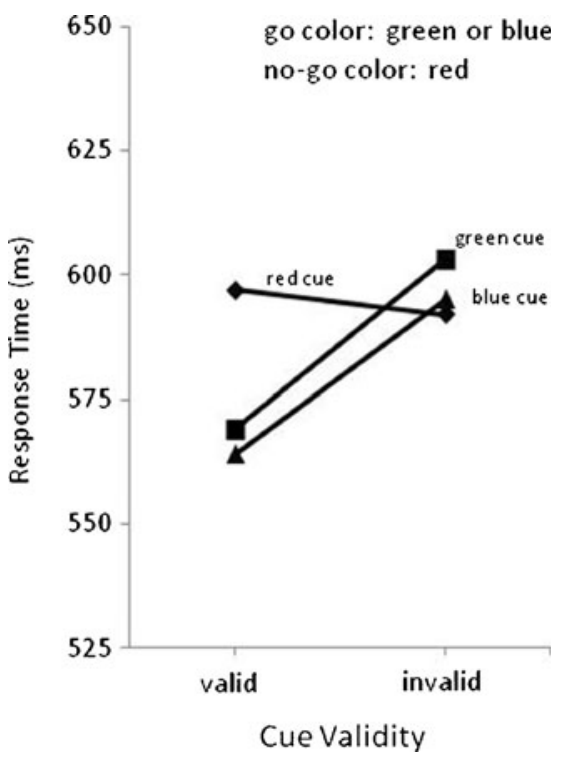

Fig. 7 Mean response time as a function of cue validity and cue color in Experiment 5. Go targets were unpredictably blue or green, while no-go targets were red cue color and cue validity, $F(2,42)=5.85, p<.01, \eta_{\mathrm{p}}{ }^{2}=$ .22. Simple-effects analyses of the effects of cue validity at each level of cue color revealed significant positive cuing effects for blue and green cues, $F(1,21)=11.99, p<.01$, $\eta_{\mathrm{p}}^{2}=.36$, and $F(1,21)=14.00, p<.01, \eta_{\mathrm{p}}{ }^{2}=.40$, respectively, while the red (no-go color) cue showed no reliable difference between valid and invalid trials, $F<1$. Replicating the results of the previous experiment, simple effects also revealed no effect of cue color on invalid trials, $F<1$, but a highly significant effect on valid trials, $F(2,42)=9.11$, $p<.001, \eta_{\mathrm{p}}^{2}=.30$. Pairwise comparisons in the valid condition revealed that red cues produced significantly longer RTs than the blue or green cues, which did not differ from one another.

A similar ANOVA was conducted on error rate. This analysis revealed no significant main effects or interactions. The error rate data are depicted in Table 1.

\section{Discussion}

The results of Experiment 5 closely replicate the results of Experiment 4 using a different color assignment. Go color cues again produced large cuing effects indicative of the capture of spatial attention. Furthermore, no-go color cues again produced similarly elevated RTs on invalid trials, along with significantly elevated RTs on valid trials, reflecting the capture of spatial attention and location-specific, feature-based inhibition, respectively. Most important, this result occurred when overall RTs were approximately 100 ms shorter than those in Experiment 4 and of a similar magnitude to those in Experiments 1 and 2, ruling out the possibility that a ceiling effect was obscuring a large positive cuing effect for the no-go color cues in Experiment 4.

One might still argue that perhaps target uncertainty produces a slowing of the specific process of disengaging attention across all cue color conditions, such that even for no-go colored cues, disengagement is not fast enough to yield negative cuing effects. There are two reasons why this account is not viable. First, if target uncertainty produces slowed disengagement across color cue conditions, then, relative to Experiments 1-3 (where target color was certain), one would expect larger cuing effects overall (see Posner, Walker, Friedrich, \& Rafal, 1984). There is simply no hint of this across experiments. Second, and more important, the fact that RTs were selectively elevated for no-go color cues on valid trials without a concurrent decrease in RTs on invalid trials is inconsistent with the disengagement account, which explicitly predicts that the two should occur in concert. This pattern, however, is exactly what is predicted by the capture-independent inhibition account. Only a selective elevation of RT particular to no-go color cues would compromise our 
conclusions, and this possibility is addressed directly in Experiment 6.

\section{Experiment 6}

Experiments 4 and 5 both support the capture-independent inhibition account and appear at odds with the speed-ofdisengagement account of contingent attentional capture. We have argued that a selective overall lengthening of RT to no-go color cues is unlikely, given the results of Experiments 1 and 2 , but a potentially important difference between those two experiments and Experiments 4 and 5 is the proportion of no-go target trials. Specifically, no-go targets appeared on only $33 \%$ of the trials in Experiments 4 and 5, whereas they appeared on $50 \%$ of the trials in Experiments 1 and 2. It is possible that no-go color cues produced a nonspatial disruption in performance when no-go targets were less frequent, such that disengagement was, in fact, fast from no-go color cues, while RT on both valid and invalid trials was elevated (i.e., line b shifted upward). This nonspatial disruption might arise from a stronger prepotent response tendency that accompanies less frequent no-go trials. To rule out this possibility and to provide a third demonstration of the critical line $d$ result, we ran an experiment in which a no-go target (blue) appeared on $50 \%$ of the trials, while go targets were unpredictably red or green, thus equating the frequency of no-go targets with that in Experiments 1 and 2, while maintaining the critical manipulation of target uncertainty.

\section{Method}

Participants Twenty undergraduate students were recruited from the Johns Hopkins University human participant pool. All were screened for normal or corrected-to-normal visual acuity and color vision. Participants were compensated for their time with extra credit toward one of several psychology courses. None of the participants had participated in any of the previous experiments.

Apparatus and stimuli The apparatus and stimuli were identical to those used in Experiment 2, with the exception that go targets were red and green, while no-go targets were blue, and that the cue colors used were red, green, and blue. In addition, no chinrest was used, and the stimuli were displayed on an Asus VE247 monitor.

Design and procedure The design and procedure were identical to those in Experiment 2, with the exception that the experiment consisted of 384 total trials and that targets were red on $25 \%$ of the trials, green on $25 \%$ of the trials, and blue (no-go) on $50 \%$ of the trials.

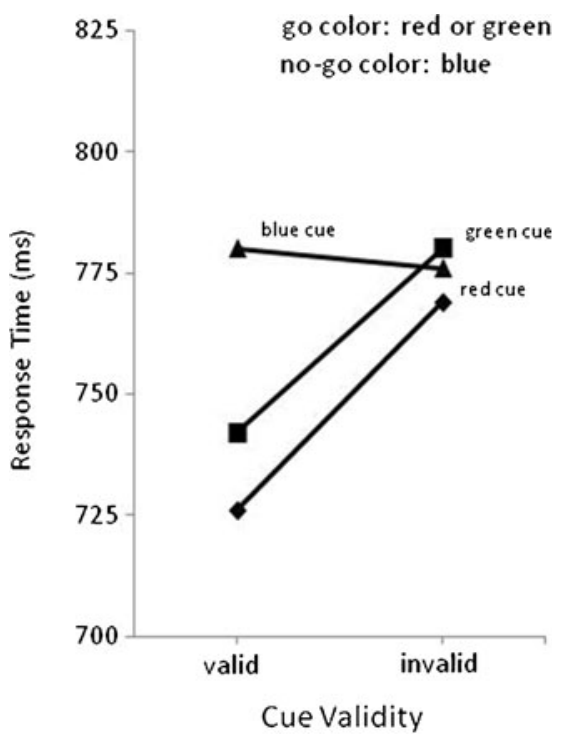

Fig. 8 Mean response time as a function of cue validity and cue color in Experiment 6. Go targets were unpredictably red or green, while nogo targets were blue

Results

Mean correct RTs for valid and invalid trials as a function of cue color are shown in Fig. 8. The data were subjected to a $3 \times 2$ ANOVA, with cue color (red, green, and blue) and cue validity (valid and invalid) as within-subjects variables. The analysis revealed main effects of cue color and cue validity, $F$ $(2,38)=3.86, p<.05, \eta_{\mathrm{p}}{ }^{2}=.17$, and $F(1,19)=14.07, p<.01$, $\eta_{\mathrm{p}}{ }^{2}=.43$, respectively, and an interaction between cue color and cue validity, $F(2,38)=4.22, p<.05, \eta_{\mathrm{p}}{ }^{2}=.18$. Simple effects analyses of the effects of cue validity at each level of cue color revealed significant positive cuing effects for red and green cues, $F(1,19)=12.10, p<.01, \eta_{\mathrm{p}}{ }^{2}=.24$, and $F(1$, $19)=9.18, p<.01, \eta_{\mathrm{p}}{ }^{2}=.19$, respectively, while the blue (nogo color) cue showed no reliable difference between valid and invalid trials, $F<1$. Simple effects also revealed no effect of cue color on invalid trials, $F<1$, but a significant effect on valid trials, $F(2,38)=4.70, p<.05, \eta_{\mathrm{p}}{ }^{2}=.20$. Pairwise comparisons in the valid condition revealed that blue cues produced significantly longer RTs than the red or green cues, which did not differ from one another.

A similar ANOVA was conducted on error rate. This analysis revealed only a main effect of validity, $F(1,19)=$ $5.90, p<.05, \eta_{\mathrm{p}}{ }^{2} .24$. The error rate data are depicted in Table 1. It should be noted that although the overall error rates are quite a bit higher than in the previous experiments (for which we have no particular explanation), the pattern of errors is similar to the pattern of RTs, indicating that the interpretation of the RT data is not compromised by a speed-accuracy trade-off. 


\section{Discussion}

The results of Experiment 6 replicate those of Experiments 4 and 5. No-go targets were presented in equal proportion to that in the first two experiments, but the manipulation of target uncertainty maintained its effect on RTs for no-go color cues; RTs were similarly elevated for go and no-go color cues on invalid trials, reflecting the capture of attention, and selectively elevated for no-go color cues on valid trials, reflecting location-specific inhibition. These results rule out the possibility that no-go color cues were, in fact, producing a flat cuing effect consistent with fast disengagement (Fig. 1, line b) that was overall elevated by a general disruption in RT caused by the relative frequency of no-go targets. Thus, the results are again at odds with the predictions of the speed-of-disengagement account of contingent attentional capture and are, instead, consistent with the capture-independent inhibition account.

\section{General discussion}

The degree to which the involuntary allocation of attention is influenced by top-down set has been a topic of debate for nearly 20 years. According to contingent attentional capture theory, the initial allocation of attention is conditioned on top-down attentional control settings, such that stimuli that do not match those settings will not elicit an involuntary attention shift. The results of the modified spatial-cuing paradigm are consistent with this theory. According to salience-based approaches, in contrast, attention is always initially allocated to salient stimuli, and the effects of topdown set simply reflect the speed with which attention is disengaged from an irrelevant stimulus. Two sources of evidence have been used to support this speed-of-disengagement account. First, the magnitude of spatial-cuing effects varies with the similarity of cues to the target (Anderson \& Folk, 2010), which, on this account, directly reflects variations in the speed of disengagement. Second, cues that do not match the target property but, instead, carry a no-go target property produce evidence of location-specific inhibition (Belopolsky et al., 2010). This inhibition has been interpreted as a marker that attention was indeed allocated to the location of nonmatching cues, which, if correct, violates the principles of the contingent attentional capture theory. However, whether an attention shift is a necessary antecedent of location-specific inhibition is an open question.

The present study was designed to explore the nature of location-specific inhibition and, more generally, to test the predictions of the speed-of-disengagement account of performance in the modified spatial-cuing paradigm. Experiments 1 and 2 verified that when target color is fixed, the magnitude of spatial-cuing effects varies as a function of cue-target similarity and cues carrying a no-go target property produce negative cuing effects reflecting location-specific inhibition. As predicted by the speed-of-disengagement account, the nogo color cues produced significantly shorter RTs than did go color cues on invalid trials and significantly longer RTs on valid trials, consistent with the very fast disengagement of captured attention. However, this pattern of results is also consistent with an account in which inhibition can be applied independently of shifts of attention. According to this captureindependent inhibition account, these same no-go color cues receive feature-based suppression as a function of their no-go status and capture attention only when they match current attentional control settings for selection. The captureindependent inhibition account thus predicts that the observed pattern of results for the no-go color cues reflects inhibition in the absence of attentional capture.

To distinguish between the speed-of-disengagement and capture-independent inhibition accounts, we tested conditions in which the no-go color cues matched the predicted attentional control settings. Under such conditions, both accounts predict that these cues will capture attention. In Experiments 3-6, when target color was unpredictable, all cues produced evidence of capture regardless of similarity to the target. In addition, Experiments 4-6 showed that cues carrying the no-go target color still produced evidence of location-specific inhibition, as indicated by selectively elevated RTs on valid trials. Most critically, this effect occurred in the absence of evidence for disengagement, as indicated by RTs on invalid trials similar to those produced by targetcolored cues. This lack of an effect on invalid trials stands in clear contrast to the robust effect observed in Experiments 1 and 2 .

The pattern of results for Experiments 3-6 violates the predictions of the speed-of-disengagement account in at least two ways. First, the disengagement account predicts that location-specific inhibition can be applied only after attention has been very quickly disengaged from a cue. However, Experiments 4-6 found that no-go color cues produced evidence of inhibition (elevated RTs on valid trials) in the absence of evidence for fast disengagement (RTs also elevated on invalid trials). Second, the disengagement account predicts that the presence of location-specific inhibition should be a function of the speed of attentional disengagement. However, the results of Experiments 3 and 4 show that it is purely a function of whether the cue color is associated with a no-go target color or not. Specifically, the fact that RTs remained elevated for invalid blue cues across the two experiments shows that the speed of disengagement remained constant, and yet the presence of location-specific inhibition varied as a function of the no-go status of the color blue. Moreover, the fact that this same pattern was evident when overall RTs were reduced in Experiment 5 rules out the possibility that no go cues produce cuing 
effects that are masked by ceiling effects. In short, the overall pattern of results shows that location-specific inhibition can be dissociated from shifts of spatial attention and is, therefore, not a reliable marker of attentional capture, as assumed by Belopolsky et al. (2010).

One could argue that the speed-of-disengagement account might be salvaged by assuming that the no-go status of the critical cues has two distinct effects on performance that, when combined, produce the pattern observed in Experiments 4-6. Specifically, the no-go status might result in the rapid disengagement of attention as predicted in Fig. 1, line b, but also produce a general elevation of RTs, relative to the other cue conditions (perhaps associated with "later" stages, such as response selection). This seems unlikely for three reasons. First, there is no evidence for a general elevation of RTs for no-go colored cues in Experiments 1 and 2. Second, one would have to argue that in Experiments 4-6, any such general effect just happens to be the exact magnitude needed to yield no difference between the cue color conditions on invalid trials. Most critically, Experiment 6 demonstrates that the critical line $d$ result still occurs when the probability of a no-go target is equated with that in Experiments 1 and 2. Thus, requiring participants to search for multiple target colors, thereby facilitating singleton detection mode, alters the pattern of RTs to no-go color cues in a way that is predicted by the capture-independent inhibition account of contingent capture and is at odds with the speed-of-disengagement account.

We propose that the most parsimonious interpretation is that the results of these experiments are consistent with the contingent attentional capture theory, according to which the degree to which a stimulus produces an involuntary shift of attention is contingent on the degree to which it matches top-down attentional control settings that manifest current behavioral goals. In Experiments 1 and 2, capture by the cue was contingent on cue-target similarity, such that cues matching the target produced maximal capture, whereas nonmatching cues produced no capture. In Experiments 36 , unpredictable target colors resulted in a top-down set for color singletons in general, resulting in capture by all color singleton cues, even those that did not match the particular target colors (Folk \& Anderson, 2010; Folk \& Remington, 2008). We conclude that location-specific inhibition in the present experiments and in Belopolsky et al. (2010) is a form of inhibition that is associated with the no-go status of the cue color and is independent of whether attention has been captured by the cue or not. This conclusion is supported by Experiments 3 and 4-6, which show the absence and presence of inhibition, respectively, even though evidence for capture was obtained for the same cues in both cases. Conversely, Experiments 1 and 4-6 show the absence and presence of capture, respectively, even though evidence for inhibition was found for the same cues in both cases. Collectively, these results provide strong evidence for a dissociation between attention shifts and location-specific inhibition.

In forwarding the speed-of-disengagement account of contingent attentional capture, Belopolsky et al. (2010) additionally argued that contingent capture effects can be explained in terms of bottom-up priming, rather than topdown attentional control settings. They showed that when top-down attentional control settings varied from trial-totrial, selectivity in attentional control was influenced more by the target on the previous trial than by current attentional control settings. We believe that bottom-up priming does not provide a complete account of selectivity in attentional selection, for several reasons. First, the target was defined as a feature singleton in their experiments, leaving open the possibility that participants adopted singleton detection mode as a strategy for identifying the target. In support of this, Lien, Ruthruff, and Johnston (2010) showed that when the target can be identified only on the basis of a particular feature, trial-by-trial adjustments in attentional control settings do, in fact, determine the selectivity in attentional selection. Second, the influence of top-down attentional control settings is still evident even when the cue does not match the target on the previous trial, although contingent capture effects are indeed stronger when the cue matches the former target color (Folk \& Remington, 2008). Third, our own results (Experiment 3 ) and previous findings (Folk \& Anderson, 2010) demonstrate attentional capture by color cues that never match the color of a target under conditions of target color uncertainty, which is at odds with the notion that selectivity in attentional control is largely a function of priming produced by previously selected targets.

If location-specific inhibition is not a form of rapidly occurring IOR that reflects recovery from capture, then what, exactly, does it represent? Sawaki and Luck (2010) provided ERP evidence that location-specific inhibition can occur for a stimulus in the absence of prior attentional capture. They and others (e.g., Lamy et al., 2004) have argued that such locationspecific inhibition reflects a form of top-down control that aids in the prevention of attentional capture. Our results suggest that the suppression of to-be-ignored stimuli can occur even when attentional capture cannot be prevented, such that two independent mechanisms of top-down control selectively influence stimulus processing.

One possibility is that both of these independent mechanisms of top-down control exert an influence at the perceptual level, with attention enhancing the representation of an object or location and location-specific inhibition suppressing that representation. By this account, the representation of an object or location can be either enhanced or suppressed, or both enhanced and suppressed simultaneously, with the outcome of stimulus selection ultimately reflecting whichever process has the stronger influence. Even though the top-down suppression may, at times, largely negate the 
benefit in stimulus processing afforded by attentional selection, the capture of attention by the object of suppression would still require the reallocation of attention, which reflects the longer RTs on invalidly cued trials for no-go color cues that occurred in Experiments 4-6. Another possibility is that the location-specific inhibition we observed is not perceptual but, instead, reflects inhibition at the level of response selection; essentially, participants experience a response incompatibility effect when a go target appears in a position formerly occupied by a cue that carries a no-go association. Such an effect of response incompatibility would need to interact with the sensory representation of the target, such that it is tied to a particular region in space. Additional research will be needed to uncover the specific relationship between attentional selection and inhibition.

\section{References}

Anderson, B. A., \& Folk, C. L. (2010). Variations in the magnitude of attentional capture: Testing a two-process model. Attention, Perception, \& Psychophysics, 72, 342-352.

Bacon, W. F., \& Egeth, H. E. (1994). Overriding stimulus-driven attentional capture. Perception \& Psychophysics, 55, 485-496.

Belopolsky, A. V., Schreij, D., \& Theeuwes, J. (2010). What is topdown about contingent capture? Attention, Perception, \& Psychophysics, 72, 326-341.

Desimone, R., \& Duncan, J. (1995). Neural mechanisms of selective visual attention. Annual Review of Neuroscience, 18, 193-222.

Eimer, M., \& Kiss, M. (2008). Involuntary attentional capture is determined by task set: Evidence from event-related brain potentials. Journal of Cognitive Neuroscience, 20, 1423-1433.

Eimer, M., \& Kiss, M. (2010). Top-down search strategies determine attentional capture in visual search: Behavioral and electrophysiological evidence. Attention, Perception, \& Psychophysics, 72, 951-962.

Folk, C. L., \& Anderson, B. A. (2010). Target uncertainty and attentional capture: Color singleton set or multiple top-down control settings? Psychonomic Bulletin \& Review, 17, 421-426.

Folk, C. L., \& Remington, R. [W.] (1998). Selectivity in distraction by irrelevant featural singletons: Evidence for two forms of attentional capture. Journal of Experimental Psychology: Human Perception and Performance, 24, 847-858.

Folk, C. L., \& Remington, R. W. (2008). Bottom-up priming of topdown attentional control settings. Visual Cognition, 16, 215-231.

Folk, C. L., Remington, R. W., \& Johnston, J. C. (1992). Involuntary covert orienting is contingent on attentional control settings.
Journal of Experimental Psychology: Human Perception and Performance, 18, 1030-1044.

Folk, C. L., Remington, R. W., \& Wright, J. H. (1994). The structure of attentional control: Contingent attentional capture by apparent motion, abrupt onset, and color. Journal of Experimental Psychology: Human Perception and Performance, 20, 317-329.

Lamy, D., Leber, A., \& Egeth, H. E. (2004). Effects of task relevance and stimulus-driven salience in feature-search mode. Journal of Experimental Psychology: Human Perception and Performance, 30, 1019-1031.

Leber, A. B. (2010). Neural predictors of within-subject fluctuations in attentional control. Journal of Neuroscience, 30, 11458-11465.

Leber, A. B., \& Egeth, H. E. (2006a). Attention on autopilot: Past experience and attentional set. Visual Cognition, 14, 565-583.

Leber, A. B., \& Egeth, H. E. (2006b). It's under control: Top-down search strategies can override attention capture. Psychonomic Bulletin \& Review, 13, 132-138.

Lien, M.-C., Ruthruff, E., Goodin, Z., \& Remington, R. W. (2008). Contingent attentional capture by top-down control settings: Converging evidence from event-related potentials. Journal of Experimental Psychology: Human Perception and Performance, 34, $509-530$

Lien, M.-C., Ruthruff, E., \& Johnston, J. C. (2010). Attentional capture with rapidly changing attentional control settings. Journal of Experimental Psychology: Human Perception and Performance, $36,1-16$.

Mack, A., \& Rock, I. (1998). Inattentional blindness. Cambridge, MA: MIT Press.

Posner, M. I. (1980). Orienting of attention. Quarterly Journal of Experimental Psychology, 32, 3-25.

Posner, M. I., Walker, J. A., Friedrich, F. J., \& Rafal, R. D. (1984). Effects of parietal injury on the covert orienting of attention. Journal of Neuroscience, 7, 1863-1874.

Sawaki, R., \& Luck, S. J. (2010). Capture versus suppression of attention by salient singletons: Electrophysiological evidence for an automatic attend-to-me signal. Attention, Perception, \& Psychophysics, 72, $1455-1470$.

Theeuwes, J. (1991). Cross-dimensional perceptual selectivity. Perception \& Psychophysics, 50, 184-193.

Theeuwes, J. (1992). Perceptual selectivity for color and form. Perception \& Psychophysics, 51, 599-606.

Theeuwes, J. (1994). Stimulus-driven capture and attentional set: Selective search for color and visual abrupt onsets. Journal of Experimental Psychology: Human Perception and Performance, 20, 799-806.

Theeuwes, J. (2004). Top-down search strategies cannot override attentional capture. Psychonomic Bulletin \& Review, 11, 65-70.

Theeuwes, J. (2010a). Top-down and bottom-up control of visual selection. Acta Psychologica, 135, 77-99.

Theeuwes, J. (2010b). Top-down and bottom-up control of visual selection: Reply to commentaries. Acta Psychologica, 135, 133-139.

Yantis, S., \& Jonides, J. (1984). Abrupt visual onsets and selective attention: Evidence from visual search. Journal of Experimental Psychology: Human Perception and Performance, 10, 601-621. 